\title{
ANÁLISE DA CONSTRUÇÃO DOS CONCEITOS DE PROPORCIONALIDADE COM A UTILIZAÇÃO DO SOFTWARE GEOPLANO VIRTUAL
}

\author{
Analysis of the construction of the concepts \\ of proportionality using the software Geoplano Virtual
}

\author{
Leandra Anversa Fioreze ${ }^{1}$. Dante Barone ${ }^{2}$. Marcus Basso ${ }^{3}$. \\ Sílvia Isaia ${ }^{4}$
}

Resumo: Este artigo apresenta resultados de uma pesquisa relacionada com a utilização de recursos digitais para a aprendizagem dos conceitos de proporcionalidade. A principal base teórica para esta análise é a Teoria dos Campos Conceituais, de Gérard Vergnaud, e a metodologia escolhida é a Engenharia Didática. Os sujeitos da pesquisa são alunos de uma oitava série de uma escola municipal situada no interior do Rio Grande do Sul. Tem-se o relato de uma das atividades com a análise das construções conceituais dos alunos utilizando o software geoplano virtual. Observou-se que, na resolução das situações, nem sempre o campo conceitual pôde ser construído de forma abrangente, evidenciando um processo de construção de conhecimentos que não se dá de forma linear.

Palavras-chave: Ensino de matemática. Ensino Fundamental. Proporcionalidade. Teoria dos campos conceituais. Aprendizagem. Tecnologia educacional.

\begin{abstract}
This article presents the results of research related to the use of digital resources for learning the concepts of proportionality. The main theoretical basis for this analysis is Conceptual Fields Theory by Gérard Vergnaud, and the methodology chosen is the Didactical Engineering. The subjects of this research were eighth-grade students from a school in a small town, Rio Grande do Sul, Brazil. There was a report of one of the activities with the analysis of students' conceptual constructions using the Virtual Geoplano software. It was observed that in the resolution of situations the conceptual field cannot not always can be made in a wide-ranging way, and that the process of knowledge making is not linear.
\end{abstract}

Keywords: Mathematics teaching. Basic Education. Proportionality. Theory of conceptual fields. Learning. Educatitonal technology.

\footnotetext{
${ }^{1}$ Centro de Ciências Naturais e Exatas (CCNE), Departamento de Matemática, Universidade Federal de Santa Maria (UFSM). Rua Dr. Liberato Vieira da Cunha, 475/402, Bairro Camobi, Santa Maria, RS, CEP 97105-090, Brasil. <leandra.fioreze@gmail.com>.

${ }^{2,3}$ Universidade Federal do Rio Grande do Sul (UFRGS), Porto Alegre, RS, Brasil.

${ }^{4}$ Centro Universitário Franciscano (UNIFRA), Santa Maria, RS, Brasil.
} 
Fioreze, L. A. et al.

\section{Introdução}

Tendo em vista que a tecnologia e, com ela, os computadores estão cada vez mais presentes no universo dos alunos e da sociedade em si, desempenhando um papel importante na vida das pessoas, neste artigo é relatada uma proposta que contempla a utilização do computador para a aprendizagem dos conceitos de proporcionalidade.

Os conceitos relacionados à proporcionalidade são fundamentais para a alfabetização matemática e estão presentes em diversas avaliações em grande escala, como no Indicador Nacional de Alfabetização Funcional (INAF), no Sistema Nacional de Avaliação da Educação Básica (SAEB) e no Exame Nacional do Ensino Médio (ENEM). Na avaliação do INAF em 2002, têm-se questões envolvendo a proporcionalidade, onde são consideradas primordiais para este indicador as percepções da relação de proporcionalidade e a capacidade de solucionar situações-problema, pois estas habilidades são extremamente pertinentes nas práticas cotidianas da sociedade (FERREIRA; GOMES, 2004). Também entre os temas e descritores da prova de Matemática do SAEB, para a $8^{a}$ série do Ensino Fundamental, encontra-se o descritor D29 (Números e Operações), 'Resolver problema que envolva variações proporcionais, diretas ou inversas entre grandezas' (INSTITUTO NACIONAL DE PESQUISAS EDUCACIONAIS, 2005).

No processo ensino-aprendizagem de Matemática, experiências com a inserção do computador demonstram que há uma mudança de status na sala de aula, pois o ensino tradicional é bastante centrado na figura do professor, que é o detentor de conhecimento; enquanto que, no ensino assistido por computador, existem possibilidades de se desenvolverem experiências de ensino centrado no aluno, mudando o foco das tarefas e responsabilidades do professor para o aluno (BASSO, 2003). Percebe-se, como observado em contato frequente com os professores que atuam no Ensino Fundamental e Médio, que a formação dos professores está longe de permitir que a tecnologia adotada seja explorada em todas as suas potencialidades. Dentre inúmeros fatores (sistema, funcionamento, estrutura física da escola etc.), a prática da informática na escola, muitas vezes, distancia-se de seu caráter pedagógico (GOMES, 2004).

A aprendizagem da matemática utilizando as tecnologias pode repetir a forma com que vinha sendo utilizada sem ela, e, neste aspecto, o estado atual da educação será mantido. Importante é o professor entender como o sujeito pensa e aprende, usando as tecnologias de forma que ele alcance bons resultados, se apoderando delas e minimizando as desconfianças com relação ao seu uso, se sentindo seguro em promover as mudanças desejadas (BASSO, 2003).

Não adianta virtualizar o ensino tradicional. A tecnologia como apoio ao ensino é limitada e até desnecessária. O que se pretende é que a tecnologia seja usada como uma ferramenta para a aprendizagem. A postura pedagógica do professor define qual a utilização será feita. (MELO NETO, 2007, p.110)

O uso dos recursos digitais para a aprendizagem dos conceitos de Matemática abre um leque de possibilidades para o planejamento das atividades do professor. Neste sentido, a preparação dos professores deve também contemplar o uso das tecnologias digitais desde a 
sua formação inicial, enfatizando as habilidades para aprender a aprender, aprender a pensar, aprender a fazer e aprender a conviver. Ou seja, esta preparação está embasada nos mesmos princípios esperados que deveriam ou deverão ocorrer na escola com a formação de crianças e adolescentes que o futuro professor encontrará. "Este educador também deveria ter a liberdade para desenvolver e colocar em prática os seus projetos, suas pesquisas, testar as suas hipóteses e refletir a partir de suas experiências" (BASSO, 2003, p. 23).

A experiência aqui relatada é parte integrante de uma pesquisa de doutoramento denominada 'Atividades digitais e a construção dos conceitos de proporcionalidade: uma análise a partir da Teoria dos Campos Conceituais'. O objetivo principal deste artigo é analisar a construção dos conceitos de proporcionalidade utilizando recursos digitais, em especial, será destacado o Geoplano Virtual. Os Campos Conceituais, de Gérard Vergnaud (1993), constituem o corpo teórico principal para a análise das construções conceituais dos alunos. Os sujeitos da pesquisa são alunos de uma oitava série situada em uma escola do interior do Rio Grande do Sul.

\section{A teoria dos campos conceituais e os softwares educativos}

A teoria dos campos conceituais tem sido bastante utilizada nas pesquisas relacionadas com a educação matemática, como se pode constatar em Barreto (2001) ou em Carraher, Carraher e Schliemann (1986).

Vergnaud (1993), ao propor estudar um campo conceitual ao invés de um conceito, está considerando que, em uma situação-problema dada, o conceito não aparece isolado. A complexidade do cenário educacional advém do fato de que muitos conceitos em matemática traçam seus sentidos utilizando uma variedade de situações, e a cada situação temos vários conceitos a serem analisados. Um campo conceitual abrange um conjunto de situações cujo domínio progressivo irá exigir uma variedade de conceitos, de procedimentos e de representações simbólicas em estreita conexão (MAGINA, 2005). Neste sentido, como os conceitos tornam-se significativos através da escolha mais adequada das situações, decorre que as situações, e não os conceitos, são a principal entrada de um campo conceitual.

Para Vergnaud (2008), dominar um campo conceitual significa saber resolver problemas em diversas situações nas quais este conceito está inserido. Analisando uma grande diversidade de comportamentos e esquemas, o pesquisador compreenderá em que consiste, do ponto de vista cognitivo, este ou aquele conceito, sendo, então, relevante ter-se um grande número de situações que darão significado aos conceitos matemáticos (GOMES, 2002).

Entre os campos conceituais evocados, as estruturas multiplicativas (e a proporcionalidade $)^{5}$ ocupam posição privilegiada, sendo consideradas como conceito-pivô no ensino da matemática e na construção das estruturas cognitivas do pensamento. É bastante avançada e reconhecida, na comunidade de pesquisadores, a classificação das relações elementares e das

\footnotetext{
${ }^{5} \mathrm{O}$ campo conceitual das estruturas multiplicativas é o conjunto das situações cuja resolução implica uma ou várias multiplicações e divisões, e o conjuntos dos conceitos e teoremas que permitem analisar estas situações: proporção simples e múltipla, função linear e n-linear, quociente e produto de dimensões, combinação linear, fração, razão, número racional, múltiplo e divisor, semelhança, dentre outros (VERGNAUD, 1993).
} 
classes de problemas elementares presentes nas situações didáticas envolvendo a proporcionalidade (VERGNAUD, 1993), constituindo-se instrumentos para a análise das situações e para a análise das dificuldades enfrentadas pelos alunos.

Com relação aos softwares educativos usando a teoria dos campos conceituais, com o objetivo de analisar a construção dos conceitos de proporcionalidade, observa-se que a maioria dos softwares destinados à educação matemática não apresenta situações que evoquem uma abrangência considerável de um campo conceitual específico (GOMES, 2004). Por exemplo, para se aprender o conceito de proporcionalidade, há a necessidade de se trabalharem diversos problemas práticos e teóricos, pois um conceito comporta várias propriedades, cuja pertinência varia de acordo com as situações a serem tratadas. Essas propriedades poderão ser compreendidas de forma imediata ou não, ou, então, futuramente, no decurso da aprendizagem.

Como nenhum software irá garantir a abrangência de todas as situações necessárias ao desenvolvimento de um conceito específico (GOMES, 2004), para a realização desta pesquisa, utilizaram-se diversos softwares como: o régua e compasso, a planilha eletrônica, o geoplano, dois objetos de aprendizagem criados pelo grupo de pesquisa Rede Interativa Virtual de Educação do Centro Universitário Franciscano (RIVED/UNIFRA), do qual um dos componentes deste artigo participou ${ }^{6}$, o vídeo 'Matemática na vida: razão e proporção ${ }^{7}$, além de objetos materiais, como maquetes, molas, moedas, folhas de papel. Para o acompanhamento das aulas e permitir a socialização, bem como a interação por meio de comentários entre aluno/aluno e aluno/pesquisador, foi criado um blog no Wordpress ${ }^{8}$.

\section{Experimentação}

As aulas da fase da experimentação foram realizadas nos meses de outubro e novembro de 2008, no turno inverso ao período de aula, totalizando oito encontros de quatro horasaula cada. Os alunos trabalharam em duplas, com a exceção de um deles, que preferiu trabalhar sozinho.

A metodologia adotada na pesquisa é a Engenharia Didática. Um dos argumentos mais favoráveis a sua adoção é a possibilidade de articular a pesquisa com a ação pedagógica, contribuindo para que esta constitua um campo de pesquisa para o professor refletir sobre sua própria prática, cooperando nas discussões que possibilitam o aprimoramento do trabalho docente ao adotar práticas pedagógicas que contribuem para uma melhoria na aprendizagem da matemática (PAIS, 2001).

Como a produção dos alunos também é objeto de análise neste trabalho, foi criada uma pasta em cada computador, para cada dupla, na qual eles salvavam suas produções e

\footnotetext{
${ }^{6}$ Os objetos de aprendizagem desenvolvidos são: A matemática nas plantas de casas e mapas e Proporcionalidade e Semelhança, sendo que este último ganhou um prêmio nacional em 2006, no concurso Rede Interativa Virtual de Educação (RIVED). Os dois objetos estão disponíveis no endereço eletrônico <http://www.unifra.br/rived>. Acesso em: 07 fev. 2013.

${ }^{7}$ Disponível em: <http://www.dominiopublico.gov.br/pesquisa/PesquisaObraForm.do>. Acesso em: 07 fev. 2013.

${ }^{8}$ Aplicativo livre e gratuito voltado para a publicação de blogs na web.
} 
respondiam em folhas alguns questionamentos. Para complementar os instrumentos, analisar os dados e validar a experiência (última etapa da engenharia didática), foram realizadas filmagens e fotografias. Também foram realizadas algumas atividades on-line, como a produção de um blog da turma e a construção dos blogs de cada dupla.

As aulas tinham a seguinte dinâmica: a partir de atividades propostas ao aluno e o envolvimento deles com estas experiências, os conteúdos eram sistematizados; ou então, os conteúdos eram introduzidos através da utilização dos softwares educativos em situações que envolviam o diálogo e a interação com o aluno por meio de questões e atividades que são entremeadas pela sistematização gradual dos conteúdos. Sempre ao término da atividade, proporcionava-se a discussão no grande grupo, com a sistematização das ideias envolvidas nas atividades, em que os alunos colocavam o seu entendimento sobre o que estava sendo tratado. O aluno, ao refletir sobre como pensou para chegar à resposta e colocar isso aos seus colegas, organiza o seu pensamento, compartilhando e socializando o resultado da produção do seu conhecimento.

\section{Atividades digitais: geoplano virtual}

Dentre algumas atividades digitais planejadas, uma delas envolve a utilização do geoplano virtual (Figura 1).

O geoplano virtual utilizado foi produzido pela Universidade de Utah dos Estados Unidos da América, disponível no National Library of Virtual Manipulative (NVLM) ${ }^{9}$. Neste site, existem vários geoplanos: quadricular (formado por quadrados), isométrico (formado por triângulos equiláteros) e circular (formado por circunferências concêntricas).

Figura 1. Software geoplano virtual.

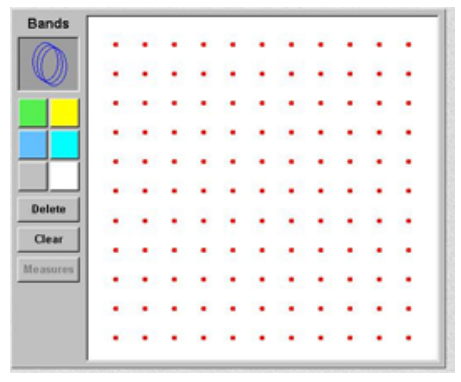

Fonte: National Library of Virtual Manipulative (NVLM) (Disponível em: <http://nlvm.usu.edu/en/nav/grade_g_4.html>. Acesso em: 07 fev. 2013.

\footnotetext{
${ }^{9}$ Estes e outros softwares educativos estão disponíveis no National Library of Virtual Manipulative, para utilização e acesso de forma pública e irrestrita para a aprendizagem escolar em nível elementar e médio. Disponível em: <http://nlvm.usu.edu/en/nav/grade_g_4.html>. Acesso em: 07 fev. 2013.
} 
Fioreze, L. A. et al.

O geoplano foi criado pelo professor Caleb Gattegno, do Instituto de Educação da Universidade de Londres. Desde seu surgimento, vários professores vêm trabalhando com ele, existindo, atualmente, uma ampla literatura, publicada, sobretudo, em revistas de Educação Matemática (KNIJNIK; BASSO; KLUSENER, 2004). Gattegno (apud KNIJNIK; BASSO; KLUSENER, 2004, p. 5) afirma que:

Todos os geoplanos têm indubitável atrativo estético e foram adotados por aqueles professores que os viram ser utilizados. Podem proporcionar experiências geométricas a crianças desde cinco anos, propondo problemas de forma, dimensão, simetria, semelhança, teoria de grupos, geometria projetiva e métrica que servem como fecundos instrumentos de trabalho, qualquer que seja o nível de ensino.

O geoplano virtual possui três versões: em inglês, francês e espanhol. A interface do software é bastante intuitiva e qualquer uma das versões é de fácil entendimento para o aluno, não necessitando de muitas explicações, pois os botões disponíveis são poucos: cores, atílios (na forma de pictograma), limpar (ao acionar este botão, o que foi construído na tela é automaticamente desfeito) e medir (área e perímetro).

\section{Análise do processo de construção dos conceitos de proporcionalidade utilizando o geoplano}

Inicialmente, os alunos foram convidados a explorar o geoplano virtual, descobrindo as ferramentas disponíveis e o seu funcionamento. Observaram que podem utilizar as cores disponíveis no software e pintaram o interior da figura construída. Após, então, se seguiu uma sequência de atividades relacionadas com a ampliação/redução de figuras geométricas.

A primeira atividade desta sequência partiu do triângulo dado, conforme Figura 2.

Figura 2. Triângulo construído no geoplano.

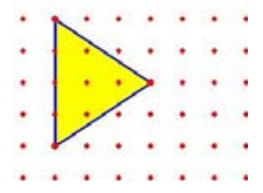

Fonte: Fioreze (2010, p. 152). 
Seguiu-se a sua ampliação, de modo que o triângulo construído ficasse semelhante ao original. Todas as duplas obtiveram êxito na questão em que a ampliação da figura se deu de modo a que todas as medidas dos lados do triângulo fossem multiplicadas por dois. Atividade semelhante foi realizada na análise prévia ${ }^{10}$, em que o êxito na questão não foi o mesmo.

$\mathrm{Na}$ segunda atividade, solicitou-se que os alunos construíssem a sequência de triângulos da Figura 3.

Figura 3. Sequência de triângulos.

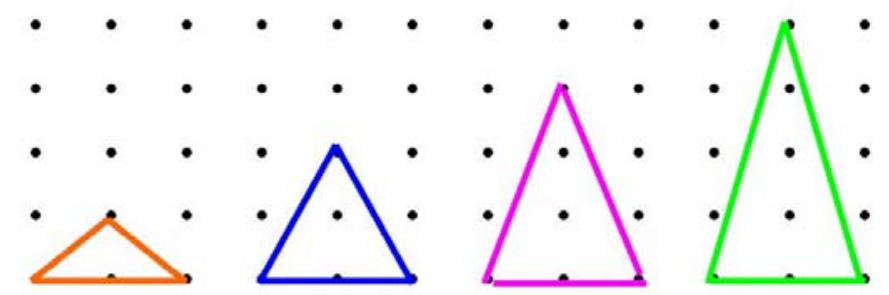

Fonte: Fioreze (2010, p. 153).

Após a construção, os alunos responderam o que eles observaram. Todas as duplas colocaram que a medida da largura manteve-se a mesma, sendo que a altura aumentou. A seguir, segue-se o questionamento: “Os triângulos são semelhantes? Por quê?". As respostas das duplas são as seguintes:

- Sim, porque em todos os triângulos aumentam $1 \mathrm{~cm}$ (aluno I);

- Não, porque não são proporcionais (dupla EF);

- Não, porque só muda a altura e deveria mudar a largura também (dupla AB).

O aluno I utilizou uma estrutura aditiva na comparação das figuras, não levando em conta que, ao se trabalhar com figuras semelhantes, deve-se utilizar um campo de saber relacionado às estruturas multiplicativas. Em uma fase inicial, os alunos apresentam, frequentemente, o raciocínio aditivo, ao invés do multiplicativo, no trato com os problemas envolvendo a proporcionalidade. Ou seja, podemos estruturar a relação construída pelo aluno sob a forma $\mathrm{a}-\mathrm{b}=\mathrm{c}-\mathrm{d}$ ao invés de $\frac{a}{b}=\frac{c}{d} \quad$ (VERGNAUD, 1993). Esta forma de pensar na resolução de problemas de proporcionalidade é chamada de raciocínio proporcional aditivo.

${ }^{10}$ Primeira etapa da Engenharia Didática, realizada com o objetivo de verificar o que os alunos sabem sobre proporcionalidade. 
Fioreze, L. A. et al.

A dupla EF incluiu a semelhança de figuras ao conjunto de problemas que envolvem a proporcionalidade. Já a dupla $\mathrm{AB}$ constatou que deveria ter havido uma mudança na largura também do triângulo para que as figuras fossem semelhantes, mas não explicou de que forma.

$\mathrm{Na}$ sequência, foi solicitado que os alunos construíssem, no geoplano, a Figura 4.

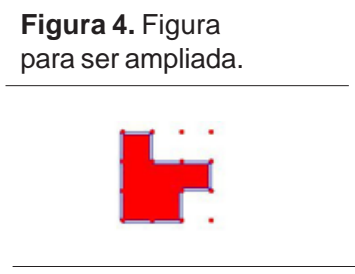

Fonte: Fioreze (2010, p. 154).

Após, eles deveriam ampliar a figura original, de forma que as medidas dos lados do polígono fossem multiplicadas por dois. As respostas dos alunos estão nas Figuras 5, 6 e 7.

Figura 5. Resposta do aluno I

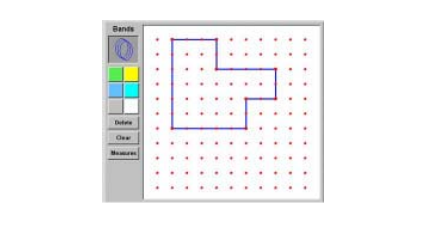

Fonte: Fioreze (2010, p. 154).
Figura 6. Resposta da dupla EF.

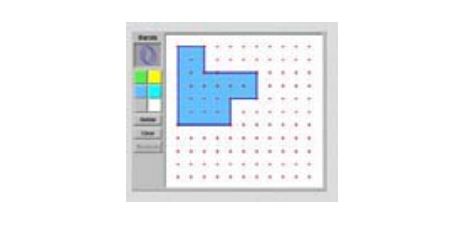

Fonte: Fioreze (2010, p. 154).
Figura 7. Resposta da dupla $A B$.

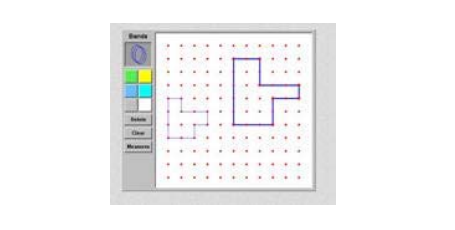

Fonte: Fioreze (2010, p. 154).

Observando as respostas, a dupla EF foi a única que conseguiu obter a figura ampliada considerando todas as medidas dos lados do polígono multiplicadas por dois.

Como esta situação envolvia uma figura mais complexa, impôs-se a necessidade de controlar diversas informações no mesmo desenho, pois um objeto geométrico tem sempre duas componentes, uma conceitual e a outra figural. A componente conceitual expressa propriedades que caracterizam uma determinada classe de objetos. Já a componente figural corresponde à imagem mental que associamos ao conceito. A harmonia entre estas duas componentes é que determina a noção correta sobre o objeto geométrico (GRAVINA, 1996).

Na Figura 4, ao ampliá-la, verificam-se dificuldades na identificação de configurações simples dentro de configurações complexas, fazendo com que condições figurais (de desenho) escapem do controle conceitual. Há a necessidade de o aluno controlar componentes conceituais, relacionados com semelhança de figuras geométricas e as condições figurais, próprias do desenho. 
A questão a seguir é dada da seguinte maneira: "Use sua criatividade e desenhe uma figura qualquer no geoplano virtual, colando no espaço abaixo". Após, solicitou-se que o aluno reduzisse a figura construída de forma que fosse semelhante à figura original. Nesta atividade, somente uma dupla não conseguiu obter êxito na questão. As Figuras 8 e 9 apresentam as respostas desta dupla (AB).

Figura 8. Figura construída pela dupla AB.

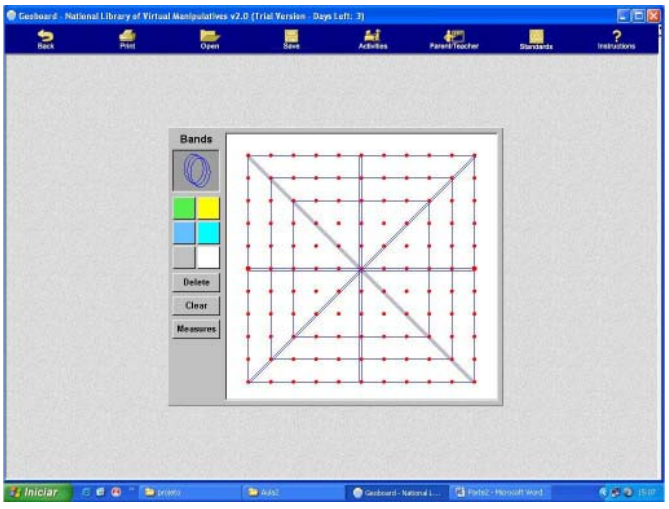

Fonte: Fioreze (2010, p. 155).
Figura 9. Figura reduzida pela dupla $A B$.

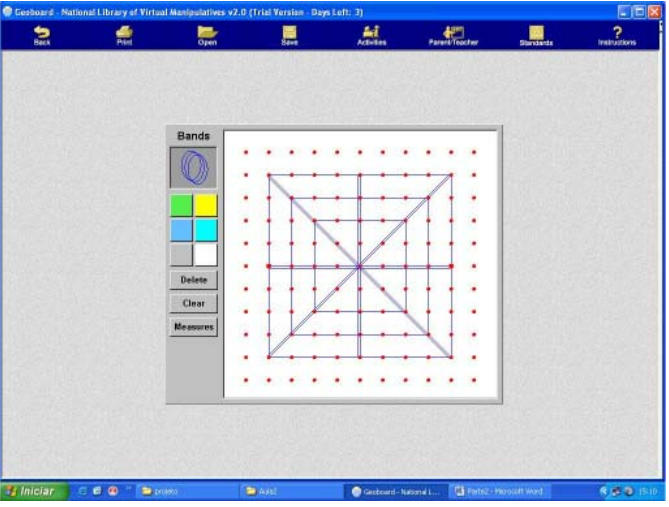

Fonte: Fioreze (2010, p. 155).

Como se observa, ou o aluno centrou-se mais na forma da figura, não estabelecendo uma relação multiplicativa entre todos os segmentos de reta que compõem as duas figuras, ou ainda tem-se, nesta situação, a emergência de um raciocínio aditivo. Como a figura construída pela dupla era mais complexa, o aluno necessita controlar diversas informações no mesmo desenho.

Com relação ao questionamento que segue: "Para você, o que são figuras semelhantes?", a dupla $\mathrm{AB}$ colocou: São tudo da mesma forma mas só que as medidas são menores. Esta dupla reduziu a classe de figuras semelhantes somente àquelas que são menores do que a figura original. Neste caso, podemos concluir que o conhecimento ainda não foi generalizado a uma classe mais ampla de situações, de modo que ele já esteja incorporado às estruturas cognitivas dos sujeitos. Em outras situações, os alunos podem estender o esquema de resolução a uma classe mais ampla do que ele seria eficaz. As respostas das outras duplas são as seguintes:

\section{Aluno I: São figuras que tenham a mesma forma e que sejam proporcionais. Dupla EF: São figuras proporcionais.}

Pode-se verificar, confrontando os resultados obtidos na análise prévia, que o aluno ampliou o conceito de semelhança de figuras planas de forma explícita, ou seja, além de 
Fioreze, L. A. et al.

explicitar este conceito, ele está mais próximo do conceito aceito pela comunidade científica. Vergnaud (1993) salienta a importância da linguagem e da utilização dos símbolos matemáticos para a conceitualização e como instrumentos para a ação, destacando que são os esquemas e as situações que lhe dão sentido.

\section{Conclusões}

Os resultados obtidos nos permitem afirmar que o envolvimento do aluno neste processo foi determinante para que a avaliação realizada na finalização do projeto fosse positiva. Para ratificar o que foi colocado, a seguir, têm-se algumas colocações dos alunos em um questionário aplicado em sala de aula, denominado de 'Avaliação do Projeto'. Uma das perguntas era: "Para você, o que mais foi significativo no projeto?". Algumas respostas foram: $A$ aprendizagem no computador, $N$ a bora das pessoas criarem seu blog, $A$ vontade dos que participaram e Que todas as pessoas se ajudavam uma às outras, onde esta última resposta apareceu duas vezes. Embora não seja o foco desta pesquisa, a motivação em aprender conteúdos utilizando o computador aparece frequentemente nas pesquisas com as tecnologias de informação e comunicação.

$\mathrm{Na}$ experimentação, objetivou-se proporcionar a participação efetiva e ativa do aluno em seu processo construtivo, valorizando os conceitos que este traz de suas experiências pessoais e de sua aprendizagem escolar, promovendo as interações entre aluno-aluno e alunoprofessor, no sentido de qualificar a aprendizagem. A falta de familiaridade dos alunos com a tecnologia foi superada com a escolha de softwares com uma interface intuitiva, não necessitando que os participantes se envolvam demasiadamente na aprendizagem do software, e sim na aprendizagem dos conceitos de proporcionalidade presentes nas atividades.

A teoria dos campos conceituais se mostrou bastante adequada para analisar as construções conceituais em que o sujeito é ativo no processo ensino-aprendizagem. Essa teoria se constituiu, também, como uma ferramenta poderosa na construção de planejamentos didáticos, pois contribuiu: no planejamento das atividades digitais e das situações de ensino, na seleção dos conceitos-chave e suas relações, assim como na análise da evolução temporal dos modelos explicativos dos sujeitos (CARVALHO JUNIOR; AGUIAR JUNIOR, 2008).

Identificamos aqui duas classes de situações em que analisamos a função adaptativa do conhecimento e suas formas nas ações dos alunos:

1. Situações em que os alunos dispunham, no seu repertório, em dado momento de seus desenvolvimentos e em certas circunstâncias, das competências necessárias ao tratamento imediato da situação;

2. Situações em que os alunos não dispunham das competências necessárias, obrigando a um tempo de reflexão e exploração, a hesitações, tentativas frustradas, levando, eventualmente, ao sucesso ou fracasso (VERGNAUD, 1993).

Como avaliado na análise prévia, os alunos, em sua maioria, não apresentaram uma conservação de forma na ampliação e redução de figuras semelhantes. Com estas atividades e outras que foram realizadas, houve uma conservação de forma na ampliação/redução de figuras semelhantes, embora não de modo a abarcar todas as situações possíveis, ou seja, este domínio de conhecimento ainda está relacionado com a situação a ser posta para os alunos. 
Análise da construção dos conceitos ...

Observou-se que, na resolução das situações com o software Geoplano, nem sempre o campo conceitual pôde ser construído de forma abrangente. Em determinadas situações, eles usavam modelos explicativos que eram mais locais; em outras, de forma mais global, evidenciando um processo de construção de conhecimentos que não se dá de forma linear.

\section{Referências}

BARRETO, I. M. A. Problemas verbais multiplicativos de quarta-proporcional: a diversidades de procedimentos de resolução. 2001. 123 f. Dissertação (Mestrado em Educação Matemática) - Pontifícia Universidade Católica, São Paulo, 2001.

BASSO, M. Espaços de aprendizagem em rede: novas orientações na formação de professores de matemática. 2003. 412 f. Tese (Doutorado em Informática na Educação) - Universidade Federal do Rio Grande do Sul, Porto Alegre, 2003.

CARRAHER, D.; CARRAHER, T. N.; SCHLIEMANN, A. D. Can mathematics teachers teach proportions? In: DAMEROW, P. et al. (Ed.). Mathematics for all. Adelaide: Unesco, 1986. p. $90-91$.

CARVALHO JÚNIOR, G. D.; AGUIAR JUNIOR, O. G. Os campos conceituais de Vergnaud como ferramenta para o planejamento didático. Caderno Brasileiro de Ensino de Física, Florianópolis, v. 25, n. 2, p. 207-227, 2008.

FERREIRA, M. C. C.; GOMES, M. L. M. O raciocínio proporcional no contexto da avaliação das habilidades matemáticas pelo $2^{\circ}$ INAF. In: FONSECA, M. C. F. R. (Org.).

Letramento no Brasil: habilidades matemáticas. São Paulo: Global, 2004. p. 127-152.

FIOREZE, L. A. Atividades digitais e a construção dos conceitos de

proporcionalidade: uma análise a partir da teoria dos campos conceituais. 2010. 245 p. Tese (Doutorado em Informática na Educação) - Universidade Federal do Rio Grande do Sul, Porto Alegre, 2010.

GOMES, A. S. et al. Avaliação de software educativo para o ensino de matemática. In: WORKSHOP DE INFORMÁTICA NA ESCOLA, 8., 2002, Florianópolis. Anais... Florianópolis: Universidade Federal de Santa Catarina, 2002. p. 1-8.

GOMES, A. S. Computadores ou computação: a noção de ubiquidade no ensino da matemática. In: ENCONTRO NACIONAL DE EDUCAÇÃO MATEMÁTICA, 8., 2004, Recife. Anais... Recife: Educandus, 2004. v. 1. p. 1-10.

GRAVINA, M. A. Geometria dinâmica: uma nova abordagem para o aprendizado da geometria. In: SIMPÓSIO BRASILEIRO DE INFORMÁTICA, 7., 1996, Belo Horizonte. Anais... Disponível em: <http://www.professores.uff.br/hjbortol/car/library/a2.doc>. Acesso em: 14 ago. 2009. 
Fioreze, L. A. et al.

INSTITUTO NACIONAL DE PESQUISAS EDUCACIONAIS. Temas e descritores da matriz de referência de matemática: SAEB / Prova Brasil. Brasília, 2005. Disponível em: $<$ http://portal.inep.gov.br/web/guest/matrizes-de-referencia-matematica-8serie > . Acesso em: 08 jun. 2007.

KNIJNIK, G.; BASSO, M. V. A.; KLUSENER, R. Aprendendo e ensinando matemática com o Geoplano. 2. ed. Ijuí: Unijuí Editora, 2004.

MAGINA, S. A Teoria dos campos conceituais: contribuições da psicologia para a prática docente. In: ENCONTRO REGIONAL DE PROFESSORES DE MATEMÁTICA, 18., 2005, Campinas. Anais... Campinas: Unicamp, 2005. Disponível em: <http:// www.ime.unicamp.br/erpm2005/anais.htm>. Acesso em: 19 mar. 2013.

MELO NETO, J. A. Tecnologia educacional: formação de professores no labirinto de ciberespaço. Rio de Janeiro: MEMVAVMEM, 2007.

PAIS, L. C. Didática da matemática: uma análise da influência francesa. Belo Horizonte: Autêntica, 2001.

VERGNAUD, G. Teoria dos campos conceituais. In: SEMINÁRIO INTERNACIONAL DE EDUCAÇÃO MATEMÁTICA DO RIO DE JANEIRO, 1., 1993, Rio de Janeiro.

Anais... Rio de Janeiro: UFRJ, 1993. p. 1-26.

Atividade humana e conceituação. Porto Alegre: GEEMPA, 2008.

Artigo recebido em 30/07/12. Aceito em 28/02/13. 